\title{
Characteristics and clinical use of ocular and cervical vestibular evoked myogenic potentials for evaluating paediatric candidates for cochlear implants
}

\author{
X-D XU ${ }^{1,2,3,4}, \mathrm{~J} \mathrm{HU}^{1}, \mathrm{Q}_{\mathrm{ZHANG}}{ }^{1}, \mathrm{Y} \mathrm{ZHANG}^{1}, \mathrm{X}-\mathrm{T} \mathrm{ZHANG}^{1}$, Y-F CHEN ${ }^{1}, \mathrm{M} \mathrm{XU}^{1}$ \\ ${ }^{1}$ Department of Otorhinolaryngology Head and Neck Surgery, Second Affiliated Hospital of Xi'an Jiaotong \\ University College of Medicine, Xi'an, ${ }^{2}$ Department of Otology and Skull Base Surgery, Eye Ear Nose \& Throat \\ Hospital, Fudan University, Shanghai, ${ }^{3}$ Shanghai Auditory Medical Center, and ${ }^{4}$ Key Laboratory of Hearing \\ Science, Ministry of Health, Shanghai, China
}

\begin{abstract}
Objective: This study aimed to define the characteristics and use of ocular and cervical vestibular evoked myogenic potentials for evaluating paediatric cochlear implant candidates.

Methods: Ocular and cervical vestibular evoked myogenic potentials of 34 paediatric cochlear implant candidates were analysed. All patients also underwent a routine audiological examination, including computed tomography.

Results: In all, 27 patients with normal inner-ear structures had absent or impaired vestibular evoked myogenic potential responses. In paediatric candidates with inner-ear malformations, ocular and cervical vestibular evoked myogenic potentials had lower thresholds and higher amplitudes. Vestibular evoked myogenic potential responses in this cohort were classified into three groups. There was significant concordance between vestibular evoked myogenic potentials and temporal bone computed tomography findings.

Conclusion: Ocular and cervical vestibular evoked myogenic potential waveforms were different in paediatric candidates with normal and abnormal inner-ear structures. Therefore, vestibular evoked myogenic potential responses can indicate temporal bone structure.
\end{abstract}

Key words: Vestibular Evoked Myogenic Potentials; Cochlear Implants; Vestibular Function Tests; Ear, Inner

\section{Introduction}

Cochlear implantation has become an important treatment for hearing loss, especially in paediatric patients with bilateral severe or profound sensorineural hearing loss (SNHL). Comprehensive pre-operative assessment of paediatric cochlear implant candidates includes otoscopy, pure tone audiometry, tympanometry, auditory brainstem response (ABR) testing, distortion product otoacoustic emission testing, high-resolution computed tomography (CT) and magnetic resonance imaging. These examinations provide inner-ear structural information, which is crucial for achieving a good surgical outcome.

Vestibular evoked myogenic potentials are induced by strong auditory or vibration stimuli and recorded from the surface of specific ocular and cervical muscles. Air-conducted sound travelling through the external auditory canal via the tympanic membrane and ossicular chain stimulates vestibular end organs (aided by endolymphatic vibration) to produce vestibular evoked myogenic potentials via specific pathways. Cervical vestibular evoked myogenic potentials are mediated by the uncrossed inhibitory vestibulocollic pathway, which begins in the saccular macula, progresses via the inferior vestibular nerve and ends in the sternocleidomastoid; they can be used to assess saccular function. ${ }^{1,2}$ Ocular vestibular evoked myogenic potentials are recorded via surface electrodes placed below the eyes when the patient looks up in response to air-conducted sound. Recent evidence strongly suggests that the utricle is the end organ involved in ocular vestibular evoked myogenic potential, regardless of the means of stimulation, although this remains controversial. ${ }^{3,4}$ Studies have demonstrated that the ocular vestibular evoked myogenic potential is elicited via a contralateral excitatory potential, and that the associated conduction pathway probably originates in the utricular macula, progresses via the superior vestibular nerve and ends in the contralateral inferior oblique muscle. $^{5-8}$ To date, potential applications of both 
ocular and cervical vestibular evoked myogenic potentials have been identified for numerous otological diseases. ${ }^{9,10}$

In theory, vestibular evoked myogenic potentials should display altered waveforms in paediatric cochlear implant candidates, especially those with inner-ear malformations, because the receptor cells and conduction pathways are influenced by abnormal conduction processes and inner-ear disease. Zhou et al. showed that cervical vestibular evoked myogenic potential testing helps in the differential diagnosis of middleear pathologies. Moreover, in patients with air-bone gaps (ABGs) seen upon pure tone audiometry, innerear structural anomalies were associated with various otological conditions. ${ }^{11}$ However, pure tone audiometry testing can be difficult in young children. Thus, it is debatable whether cervical vestibular evoked myogenic potential testing can form part of the pre-operative paediatric examination. Moreover, it is unknown whether ocular and cervical vestibular evoked myogenic potentials have similar diagnostic capabilities.

This study combined ocular and cervical vestibular evoked myogenic potential testing to identify the typical patterns of evoked potential changes in paediatric cochlear implant candidates and investigated the value of this approach in the differential diagnosis of disorders affecting the inner-ear structure.

\section{Materials and methods}

\section{Participants}

A retrospective study of paediatric cochlear implant candidates treated in the Department of Otorhinolaryngology Head and Neck Surgery, Second Affiliated Hospital of Xi' an Jiaotong University College of Medicine, from January 2012 to December 2015 was performed. A total of 34 patients (17 girls and 17 boys) with a mean age of $5.6 \pm 2.0$ years (range 3-12 years) were recruited. Twenty healthy paediatric volunteers with a mean age of $6.45 \pm 2.19$ years (range $4-10$ years; 11 girls and 9 boys) were recruited to serve as controls. All children underwent otoscopy, pure tone audiometry and tympanometry analyses. Ocular and cervical vestibular evoked myogenic potentials were assessed in 32 healthy ears.

This study was approved by the institutional review board of the Second Affiliated Hospital of Xi'an Jiaotong University College of Medicine. Guardians provided informed consent for their child's participation.

\section{Experimental procedures}

A medical history was taken for each patient, and there were no reports of any other previous ear disorder. Routine examinations including otoscopy, pure tone audiometry, tympanometry, ABR, distortion product otoacoustic emission and CT were performed if possible. The auditory steady-state response was assessed in children who were too young to cooperate with pure tone audiometry testing.
In a soundproof examination room, ocular and cervical vestibular evoked myogenic potentials were recorded in response to air-conducted sound with a $500-\mathrm{Hz}$ short tone burst (rise and fall times $=1 \mathrm{~ms}$, plateau time $=2 \mathrm{~ms}$ ) transmitted through a calibrated earphone. The electromyography signal from the stimulated side was amplified using an ICS Chartr EP analyser (GN Otometrics, Taastrup, Denmark), and the bandpass filter was set to $0.001-1 \mathrm{kHz}$. The responses to 2 sets of 50 stimuli were averaged separately. A stimulus of $131 \mathrm{~dB}$ SPL was used as the default starting intensity to check whether vestibular evoked myogenic potentials could be elicited for each patient and to assess the waveforms. The stimulus intensity was then decreased or increased in 5-dB SPL steps depending upon the presence or absence, respectively, of vestibular evoked myogenic potentials. The lowest stimulus intensity to elicit a clear, reproducible biphasic wave was recorded (in dB SPL).

Ocular vestibular evoked myogenic potential testing was performed with participants in a supine position. An active electrode was then placed $1 \mathrm{~cm}$ below the lower lid of each eye in line with the pupil, a reference electrode was placed below the active electrode and a ground electrode was placed on the midline of the forehead; an inter-electrode resistance of less than $5 \mathrm{k} \Omega$ was confirmed. Each participant was asked to look upwards upon hearing a sound from the insert earphone. ${ }^{5}$ Attention-attracting tools (e.g. toys) were used for children who could not follow this instruction because they were either too young or could not hear the sound.

Cervical vestibular evoked myogenic potential testing was similarly performed with participants in a supine position. An active electrode was placed in the middle of the sternocleidomastoid muscle, a reference electrode was placed above the sternoclavicular joint and a ground electrode was placed on the midline of the forehead; an inter-electrode resistance of less than $5 \mathrm{k} \Omega$ was confirmed. Each participant was instructed to raise her or his head off the pillow to activate the sternocleidomastoid muscle electrode upon hearing tone bursts through the insert earphone. ${ }^{12}$ Attentionattracting tools were used for children who were unable to follow these instructions.

\section{Analysis of vestibular evoked myogenic potential parameters}

Vestibular evoked myogenic potentials were considered suitable when a reproducible short-latency biphasic wave was elicited; unrecognisable or unrepeatable waveforms were rejected. Response rates were calculated for both ocular and cervical vestibular evoked myogenic potentials.

Vestibular evoked myogenic potential thresholds were measured as described; amplitudes and latencies of ocular and cervical vestibular evoked myogenic potentials were measured using a 131-dB SPL stimulus. P1 and $\mathrm{N} 1$ are the first positive and negative peaks, respectively. The following parameters were 
recorded: the P1 and N1 latencies (in ms), defined as the interval between $0 \mathrm{~ms}$ and the corresponding maximal peak; the inter-peak latencies (in ms), calculated as the interval between $\mathrm{P} 1$ and $\mathrm{N} 1$; and the amplitude (in $\mu \mathrm{V}$ ), defined as the vertical distance between the $\mathrm{P} 1$ and $\mathrm{N} 1$ peaks.

\section{Statistical analysis}

Data was analysed using IBM SPSS Statistics software version 19.0 (Armonk, New York, USA). $\chi^{2}$ tests were used to compare between-group response rates; Student's $t$-tests were used to compare vestibular evoked myogenic potential thresholds, P1 and N1 latencies, inter-peak latencies and amplitudes; and Cohen's $\kappa$ and McNemar tests were used to determine concordance between the different examinations. A $p$ value of less than 0.05 was considered statistically significant.

\section{Results}

Computed tomography imaging of inner-ear structures All 34 patients (68 ears) had shown a poor response to sound from a very young age, but with no other reported ear disorder. Temporal bone CT showed that 27 patients (54 out of 68 ears) had normal middleand inner-ear structures (normal structure group); 6 patients (10 ears) had large vestibular aqueduct syndrome and 1 patient ( 2 ears) had bilateral Mondini dysplasia with enlarged vestibular aqueducts (a total of 12 ears; abnormal structure group 1). In the remaining two ears (one with moderate and the other with mild hearing loss), temporal bone imaging showed large vestibular aqueduct syndrome; these two ears were added to abnormal structure group 1 to form abnormal structure group 2 . The diagnostic criteria for large vestibular aqueduct syndrome were those described by Valvassori and Clemis. ${ }^{13}$ Briefly, a vestibular aqueduct was considered to be enlarged if the midpoint width was larger than $1.5 \mathrm{~mm}$ or wider than the posterior semicircular canal diameter.

\section{Routine audiological evaluations}

The findings of routine audiological evaluations are summarised in Table I. All 68 ears had normal external canals and tympanic membranes. However, not all tests were completed successfully because of poor patient cooperation. Audiological examination findings showed severe or profound SNHL in the normal structure group and abnormal structure group 1 with no response to distortion product otoacoustic emissions (DPOAEs). Of the two ears not included in the normal structure group or abnormal structure group 1, one had moderate hearing loss with a type $\mathrm{C}$ curve in the emittance testing and the other had mild hearing loss and low-frequency loss of DPOAEs. In the normal structure group and abnormal structure group 1, 30 type A (normal) curves and 16 abnormal curves were observed for 66 ears. There was no significant relationship between inner-ear structure and routine audiological findings.

\section{Ocular and cervical vestibular evoked myogenic potential results}

All children underwent ocular and cervical vestibular evoked myogenic potential testing. In the normal structure group, ocular vestibular evoked myogenic potentials were absent in 19 ears and present in the remaining 35 ears. Similarly, cervical vestibular evoked myogenic potentials were absent in 16 patients and present in 36: 1 patient's parents did not consent to this test for personal reasons. Ocular and cervical vestibular evoked myogenic potentials were present in all patients in abnormal structure group 1, except for one with bilateral large vestibular aqueduct syndrome who refused to undergo cervical vestibular evoked myogenic potential testing. The results of ocular and cervical vestibular evoked myogenic potential testing are summarised in Table II.

In the normal structure group, the response rate was 65 per cent (35 out of 54 ears) for ocular vestibular evoked myogenic potentials and 69 per cent (36 out of 52) for cervical vestibular evoked myogenic potentials. However, in abnormal structure group 1 (comprising children with large vestibular aqueduct syndrome and Mondini dysplasia), response rates were 100 per cent for both ocular and cervical vestibular evoked myogenic potentials. Ocular vestibular evoked myogenic potential response rates were significantly different between the normal structure group and abnormal structure group 1 and between the normal structure group and abnormal group $2(p<0.05)$. Cervical

\begin{tabular}{|c|c|c|c|c|c|c|c|c|c|c|}
\hline & ORY BR & STEM & PONSE, D & $\begin{array}{r}\text { ORTIO } \\
\text { FIN }\end{array}$ & $\begin{array}{l}\text { TABLE } \\
\text { RODUC } \\
\text { NGS BY }\end{array}$ & $\begin{array}{l}\text { TOACOUST } \\
\text { OUP }\end{array}$ & EMISS & NS AND & MPANON & \\
\hline Group & Ears $(n)$ & & ABR & & & DPOAE & & & mpanometry & \\
\hline & & Normal & Abnormal & DNT & Normal & Abnormal & DNT & Normal & Abnormal & DNT \\
\hline $\mathrm{N}$ & 54 & 0 & 52 & 2 & 0 & 52 & 2 & 24 & 12 & 18 \\
\hline A1 & 12 & 0 & 12 & 0 & 0 & 9 & 3 & 6 & 4 & 2 \\
\hline A2 & 14 & 1 & 13 & 0 & 0 & 10 & 4 & 7 & 5 & 2 \\
\hline
\end{tabular}

$\mathrm{ABR}=$ auditory brainstem response; $\mathrm{DPOAE}=$ distortion product otoacoustic emission; $\mathrm{DNT}=$ did not test; $\mathrm{N}=$ normal structure group; $\mathrm{A} 1=$ abnormal structure group $1 ; \mathrm{A} 2=$ abnormal structure group 2 


\begin{tabular}{lccccccc}
\multicolumn{1}{c}{ TABLE II } \\
OCULAR AND CERVICAL VESTIBULAR EVOKED MYOGENIC POTENTIAL FINDINGS BY GROUP
\end{tabular}

oVEMP $=$ ocular vestibular evoked myogenic potentials; $\mathrm{cVEMP}=$ cervical vestibular evoked myogenic potentials; DNT $=$ did not test; $\mathrm{N}=$ normal structure group; $\mathrm{A} 1=$ abnormal structure group $1 ; \mathrm{A} 2=$ abnormal structure group 2

\begin{tabular}{|c|c|c|c|c|c|c|}
\hline & 3ULAR $\mathrm{E}$ & ED MYOGENIC PO & $\begin{array}{r}\text { TABLE I } \\
\text { ГIALS: ASSOCIA }\end{array}$ & NS BETW & THRESHOLDS AN & PLITUDES \\
\hline \multirow[t]{2}{*}{ Group } & \multicolumn{3}{|c|}{ oVEMP } & \multicolumn{3}{|c|}{ cVEMP } \\
\hline & Ears $(n)$ & Threshold (dB SPL) & Amplitude $(\mu \mathrm{V})$ & Ears $(n)$ & Threshold (dB SPL) & Amplitude $(\mu \mathrm{V})$ \\
\hline $\mathrm{N}$ & 35 & $125.0 \pm 4.8$ & $5.5 \pm 3.5$ & 36 & $118.8 \pm 5.4$ & $299.9 \pm 179.7$ \\
\hline A1 & 12 & $116.0 \pm 4.3^{*}$ & $13.4 \pm 9.3^{\dagger}$ & 10 & $111.5 \pm 7.6^{*}$ & $407.2 \pm 175.3$ \\
\hline A2 & 14 & $114.6 \pm 5.7^{\ddagger}$ & $15.6 \pm 10.2$ & 12 & $110.2 \pm 7.6^{\ddagger}$ & $410.5 \pm 180.1$ \\
\hline
\end{tabular}

${ }^{*} \mathrm{~A} 1$ group $v s \mathrm{~N}$ group, Student's $t$-test $p<0.01 .{ }^{\dagger} \mathrm{A} 1$ group $v s \mathrm{~N}$ group, Student's $t$-test $p<0.05 .{ }^{\star} \mathrm{A} 2$ group $2 v s \mathrm{~N}$ group, Student's $t$-test $p<$ 0.01. oVEMP $=$ ocular vestibular evoked myogenic potentials; $\mathrm{cVEMP}=$ cervical vestibular evoked myogenic potentials; $\mathrm{DNT}=$ did not test; $\mathrm{N}=$ normal structure group; $\mathrm{A} 1=$ abnormal structure group $1 ; \mathrm{A} 2=$ abnormal structure group 2

vestibular evoked myogenic potential response rates were also significantly different between the normal structure group and abnormal structure group 2, although not between the normal structure group and abnormal structure group $1(p=0.052)$.

Vestibular evoked myogenic potential parameters in ears with normal and abnormal inner-ear structures are summarised in Table III. Threshold values were significantly different for ears with different inner-ear structures $(p<0.01)$. The amplitudes of ocular vestibular evoked myogenic potentials differed significantly with respect to inner-ear structure (i.e. normal $v s$ abnormal), whereas those of cervical vestibular evoked myogenic potentials did not. Scatter plots illustrating differences in vestibular evoked myogenic potentials between ears with normal $v s$ abnormal inner-ear structures are shown in Figure 1. It is apparent that the scatterplots have different shapes for different patient groups. However, the inter-peak latency and P1 and N1 latencies of ocular and cervical vestibular evoked myogenic potentials showed no obvious patterns.

\section{Concordance between ocular and cervical vestibular evoked myogenic potentials and computed tomography findings}

Based on the analysis described above, three types of vestibular evoked myogenic potential results could be classified (shown in Figure 2): type 1, absent (no waveforms); type 2, impaired (characterised by threshold elevation and a reduced amplitude); and type 3, sensitivity (characterised by threshold reduction and an increased amplitude).

To establish a classification standard for types 2 and 3 vestibular evoked myogenic potential, 32 healthy ears were tested. For the ocular vestibular evoked myogenic potential, the median threshold was $116 \mathrm{~dB}$ SPL, and the mean amplitude was $8.57 \mu \mathrm{V}$; for the cervical vestibular evoked myogenic potential, these parameter were $111 \mathrm{~dB}$ SPL and $360.88 \mu \mathrm{V}$, respectively. By comparing the respective values in patients and healthy controls, above median threshold and mean amplitude values were chosen as classification standards for both ocular and cervical vestibular evoked myogenic potentials (as shown in Figure 3).

The classification of the ocular and cervical vestibular evoked myogenic potentials is summarised in Table IV. Cohen's $\kappa$ coefficients and paired McNemar tests showed significant concordance between cervical vestibular evoked myogenic potentials and temporal bone CT findings. A similar trend was found between the ocular vestibular evoked myogenic potentials and CT findings, regardless of whether the normal structure group was compared with abnormal structure group 1 or abnormal structure group 2.

\section{Discussion}

Paediatric severe or profound SNHL accompanied by inner-ear malformation is not rare. The ocular and cervical vestibular evoked myogenic potential pathways include the external auditory canal, ossicular chain and endolymph. Abnormal pathway structures are revealed by changes to waveforms. In this study, two vestibular evoked myogenic potentials triggered via air-conducted sound were used to assess otolithic function and conduction pathways. Concordance between vestibular evoked myogenic potentials and the results of routine pre-operative evaluations for cochlear 

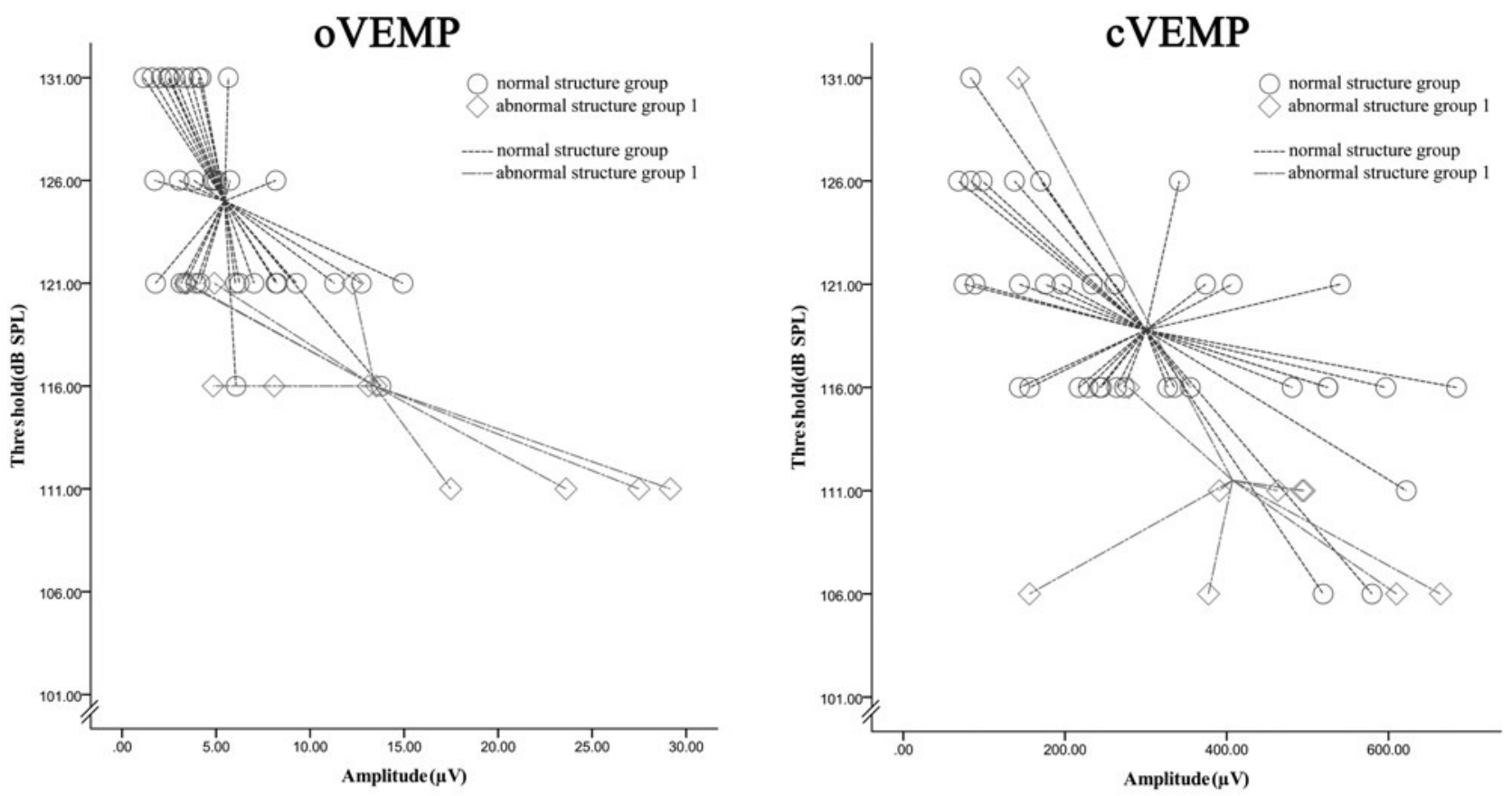

FIG. 1

Scatter plots showing ocular and cervical vestibular evoked myogenic potentials. The central point was tagged for each group to display between-group differences. $\mathrm{N}=$ normal structure group; $\mathrm{A} 1=$ abnormal structure group 1; $\mathrm{A} 2$ = abnormal structure group 2

implantation was investigated. Fourteen out of 68 ears ( 21 per cent) in the present study had inner-ear malformations; this finding is consistent with those of Van
Wermeskerken et al. (20 per cent). ${ }^{14}$ Routine audiological evaluation of paediatric candidates showed abnormal $\mathrm{ABR}$ and distortion product otoacoustic

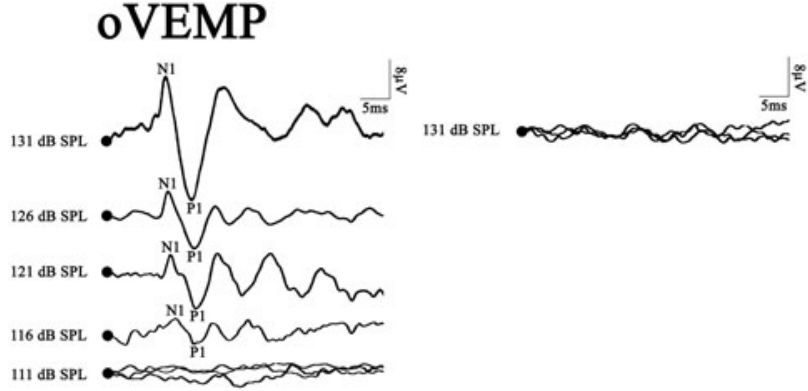

Normal

cVEMP

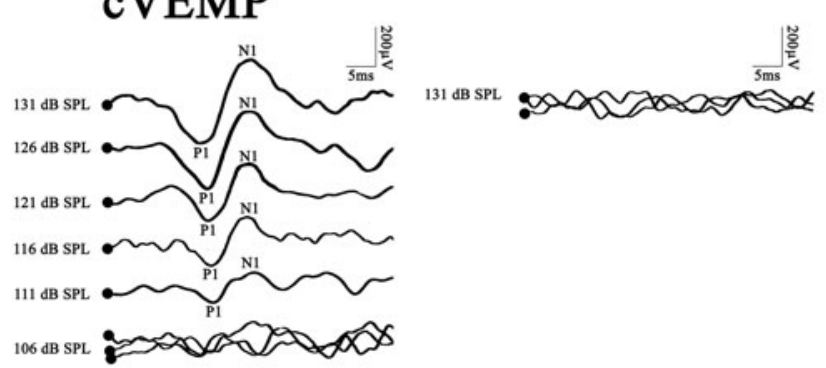

Normal

Type 1

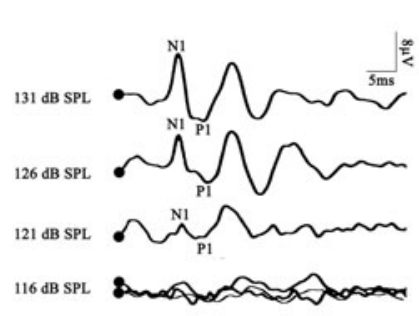

Type 2
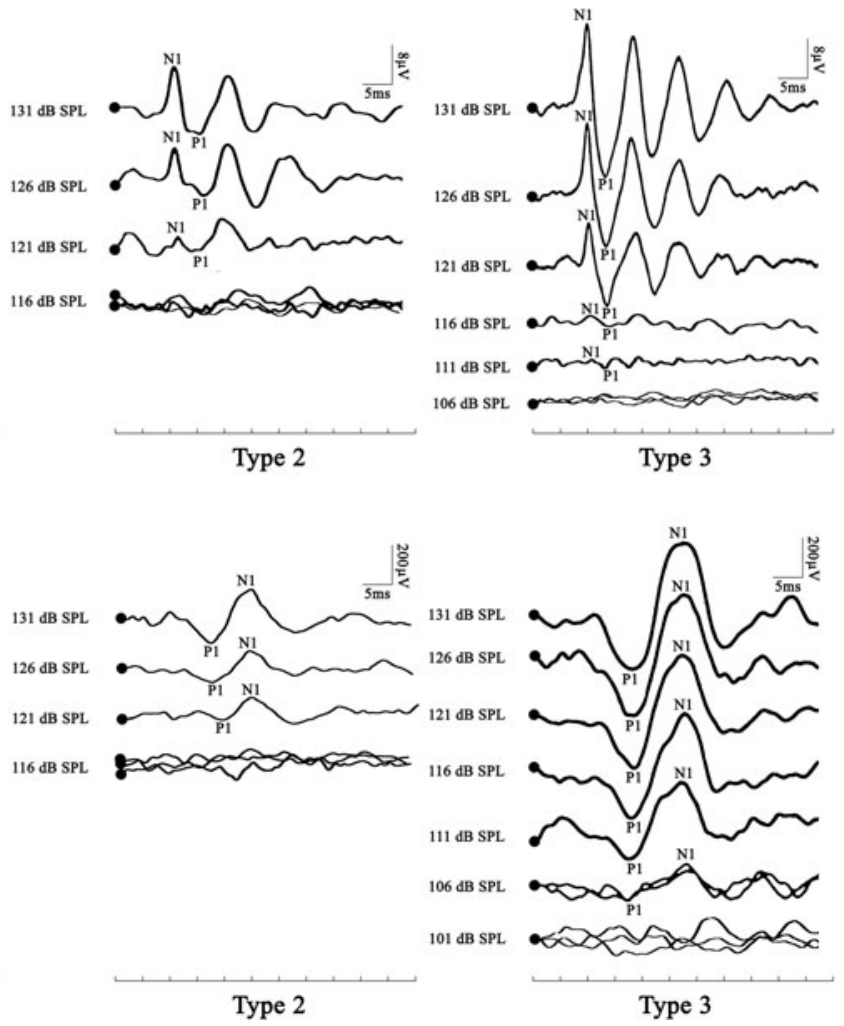

FIG. 2

Graphs showing typical waveforms for a normal healthy ear and for ears with types 1, 2 and 3 ocular (a) and cervical (b) vestibular evoked myogenic potentials. Type 1, absence; type 2, impaired; type 3, sensitivity. P1 = first positive peak; N1 = first negative peak 


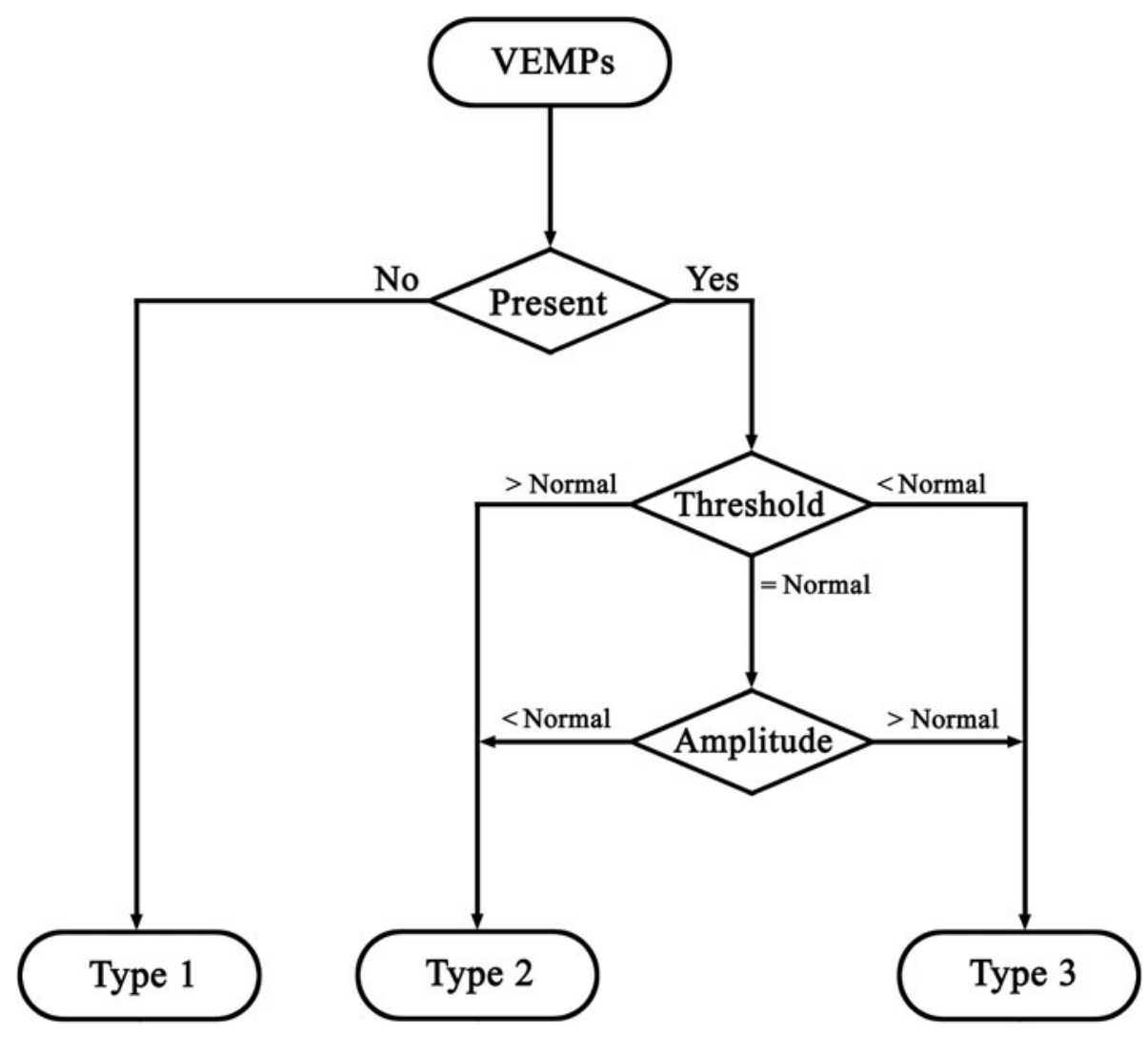

FIG. 3

Flow chart showing the development of a temporary standard used to classify ocular and cervical vestibular evoked myogenic potentials (VEMPs) in this study.

TABLE IV

CONCORDANCE BETWEEN VESTIBULAR EVOKED MYOGENIC POTENTIALS AND COMPUTED TOMOGRAPHY FINDINGS

\begin{tabular}{|c|c|c|c|c|c|c|c|c|c|c|}
\hline \multirow[t]{2}{*}{ Group } & \multicolumn{5}{|c|}{ oVEMP } & \multicolumn{5}{|c|}{ cVEMP } \\
\hline & Ears $(n)$ & Types $1 \& 2$ & Type 3 & Cohen's $\kappa^{*}$ & $p$ value $^{\dagger}$ & Ears $(n)$ & Types $1 \& 2$ & Type 3 & Cohen's $\kappa^{*}$ & $p$ value $^{\dagger}$ \\
\hline $\mathrm{N}$ & 54 & 53 & 1 & - & - & 52 & 49 & 3 & - & - \\
\hline A1 & 12 & 6 & 6 & 0.575 & 0.125 & 10 & 2 & 8 & 0.713 & 1.000 \\
\hline A2 & 14 & 6 & 8 & 0.637 & 0.125 & 12 & 2 & 10 & 0.752 & 1.000 \\
\hline
\end{tabular}

${ }^{*}$ A value of $>0.4$ indicates concordance and a value of $>0.75$ indicates very strong concordance. ${ }^{\dagger}$ In McNemar testing, $p>0.05$ indicates no difference between vestibular evoked myogenic potential and CT findings. oVEMP $=$ ocular vestibular evoked myogenic potentials; cVEMP = cervical vestibular evoked myogenic potentials; $\mathrm{N}=$ normal structure group; $\mathrm{A} 1$ = abnormal structure group $1 ; \mathrm{A} 2=\mathrm{abnormal}$ structure group 2

emission results for the normal structure group and abnormal group 1. In abnormal structure group 2, one ear affected by large vestibular aqueduct syndrome had a normal ABR; however, this ABR is likely to become abnormal with disease progression. Tympanometry results for this ear were inconclusive and the inner-ear structure did not show a clear correlation with any test result.

However, vestibular evoked myogenic potentials may be different with the different tests described above. Sound travels through the external auditory canal via the tympanic membrane and ossicular chain to stimulate vestibular end organs (aided by endolymphatic vibration) and produces air-conducted sound vestibular evoked myogenic potentials through specific pathways. After researched the pure tone audiometry of patients with ABGs, Zhou et al. reported no clear correlations between tympanometry, acoustic reflex and CT findings for the middle and inner ear. ${ }^{11}$ In contrast, cervical vestibular evoked myogenic potentials have been used for differential diagnosis in patients with ABGs and/or conductive components associated with various otological conditions. Abnormalities in the conductive process may change vestibular evoked myogenic potentials, thus extending their clinical applications. In the present study, the following vestibular evoked myogenic potential characteristics were observed. Firstly, ocular and cervical vestibular 
evoked myogenic potentials were elicited for all patients with inner-ear malformations (abnormal structure group 2), and the response rates were significantly higher than in the normal structure group. Secondly, ocular vestibular evoked myogenic potential thresholds were much lower and their amplitudes were much broader in patients with inner-ear malformations (abnormal structure group 2) than in the normal structure group. Similar results were obtained for cervical vestibular evoked myogenic potentials.

The cochlea and vestibule share a continuous membranous structure and have similar receptor cells; therefore, inner-ear disease can affect both hearing and balance. The utricle and saccule, which are both affected by inner-ear diseases, serve as receptors for ocular and cervical vestibular evoked myogenic potentials. The type of change in vestibular evoked myogenic potentials can indicate differences in inner-ear disease severity affecting the utricle and saccule.9,10,15 For example, severe inner-ear disease may render receptor cells of the utricle and saccule unable to respond to stimulation. Thus, the typical waveforms of ocular and cervical vestibular evoked myogenic potentials are not elicited, and there is a consequent decline in response rate (type 1 pattern). In contrast, relatively minor damage may impair the formation of waveforms: in this case, the otolith organ stimulation effectively elicits both ocular and cervical vestibular evoked myogenic potentials, although with abnormally higher thresholds and lower amplitudes (type 2 pattern). Type 3 ocular and cervical vestibular evoked myogenic potentials have a different pattern, comprising abnormally low thresholds and broader amplitudes. In this study, types 1 and 2 vestibular evoked myogenic potentials were highly concordant with $\mathrm{CT}$ results of candidates with normal inner-ear structures, and type 3 waveform patterns were highly concordant with CT results of candidates with inner-ear malformations. Ocular and cervical vestibular evoked myogenic potentials were more excitable and sensitive in patients with inner-ear malformations. Sheykholeslami et al. and Merchant et al. reported the same phenomenon in patients with large vestibular aqueduct syndrome. ${ }^{16,17}$ These authors suggested that a close relationship exists between the sensitive cervical vestibular evoked myogenic potential and the so-called third-window effect caused by inner-ear malformation. A type 3 waveform pattern indicating vestibular evoked myogenic potential sensitivity was also found in superior semicircular dehiscence syndrome, which is proposed to be another thirdwindow condition. ${ }^{18,19}$ A similar mechanism may also exist in Mondini dysplasia patients because increased endolymphatic fluid vibration in cochlear and vestibular dysplasia might promote vestibular evoked myogenic potential sensitivity. However, further research is necessary to fully understand endolymphatic fluid hydrodynamics in inner-ear malformations.

To assess whether vestibular evoked myogenic potentials can be used to identify inner-ear structures, vestibular evoked myogenic potentials were classified as type 1, 2 or 3 using a temporary classification standard based on data for vestibular evoked myogenic potential parameters in normal children. In this study, vestibular evoked myogenic potential data were consistent with CT findings. Although the accuracy of ocular and cervical vestibular evoked myogenic potential data for diagnosing inner-ear structures needs to be improved, their clinical value was clearly demonstrated. Analysis of larger patient cohorts may define the normal range of various vestibular evoked myogenic potential parameters. The most useful are likely to be the threshold and the amplitude.

\section{- Vestibular evoked myogenic potentials were measured in paediatric cochlear implant candidates}

- Ocular and cervical vestibular evoked myogenic potential waveforms differed significantly between those with normal and abnormal inner-ear structures

- Vestibular evoked myogenic potentials correlated closely with temporal bone computed tomography findings

- Vestibular evoked myogenic potentials may be useful for diagnosing inner-ear disease

Two cochlear implant candidates in this study had bilateral large vestibular aqueduct syndrome: one had profound SNHL in the right ear and moderate hearing loss in the left, and the other had profound SNHL in the right ear and mild hearing loss in the left. In both patients, the vestibular evoked myogenic potentials in the non-SNHL ear had reduced thresholds and increased amplitudes that were identical to those of the contralateral ears with profound SNHL. These data suggest that concordance between vestibular evoked myogenic potentials and CT findings may not be limited to children with severe or profound SNHL.

\section{Conclusion}

Inner-ear malformation is common in paediatric cochlear implant candidates. In this study, ocular and cervical vestibular evoked myogenic potential waveforms showed a regular pattern of changes in children with inner-ear malformations that were significantly different from those of paediatric cochlear implant candidates with normal inner-ear structures. Vestibular evoked myogenic potentials correlated closely with inner-ear structure as indicated by temporal bone $\mathrm{CT}$. In addition to providing a method for objectively assessing otolithic function, vestibular evoked myogenic potentials may have a future role in diagnosing inner-ear disease. 


\section{Acknowledgements}

This study was supported by the National Natural Science Foundation of China (81541040, 81670945 to QZ, $3013300 / \mathrm{C} 170703$ to MX), the Shaanxi Major International Cooperative Project of China (2013KW-28 to MX) and the Key Clinical Program of the Second Affiliated Hospital of Xi'an Jiaotong University College of Medicine (SF1315(1) to QZ).

\section{References}

1 Colebatch JG, Halmagyi GM, Skuse NF. Myogenic potentials generated by a click-evoked vestibulocollic reflex. J Neurol Neurosurg Psychiatry 1994;57:190-7

2 Halmagyi GM, Colebatch JG. Vestibular evoked myogenic potentials in the sternomastoid muscle are not of lateral canal origin. Acta Otolaryngol Suppl 1995;520(Pt 1):1-3

3 Curthoys IS. A critical review of the neurophysiological evidence underlying clinical vestibular testing using sound, vibration and galvanic stimuli. Clin Neurophysiol 2010;121:132-44

4 Govender S, Rosengren SM, Todd NP, Colebatch JG. Ocular vestibular evoked myogenic potentials produced by impulsive lateral acceleration in unilateral vestibular dysfunction. Clin Neurophysiol 2011;122:2498-504

5 Iwasaki S, Chihara Y, Smulders YE, Burgess AM, Halmagyi GM, Curthoys IS et al. The role of the superior vestibular nerve in generating ocular vestibular-evoked myogenic potentials to bone conducted vibration at Fz. Clin Neurophysiol 2009; 120:588-93

6 Todd NP, Rosengren SM, Colebatch JG. Ocular vestibular evoked myogenic potentials (OVEMPs) produced by impulsive transmastoid accelerations. Clin Neurophysiol 2008;119: $1638-51$

7 Murofushi T, Nakahara H, Yoshimura E, Tsuda Y. Association of air-conducted sound oVEMP findings with cVEMP and caloric test findings in patients with unilateral peripheral vestibular disorders. Acta Otolaryngol 2011;131:945-50

8 Curthoys IS, Vulovic V, Manzari L. Ocular vestibular-evoked myogenic potential (oVEMP) to test utricular function: neural and oculomotor evidence. Acta Otorhinolaryngol Ital 2012; 32:41-5

$9 \mathrm{Xu}$ XD, Zhang Q, Hu J, Zhang Y, Chen YF, Zhang XT et al. The hidden loss of otolithic function in children with profound sensorineural hearing loss. Int J Pediatr Otorhinolaryngol 2015;79: $852-7$
$10 \mathrm{Xu}$ XD, Ding CR, Yu J, Han Z, Gu J, Gao N et al. The hidden dysfunction of otolithic organs in patients with profound sensorineural hearing loss. Hear Res 2016;331:41-6

11 Zhou G, Poe D, Gopen Q. Clinical use of vestibular evoked myogenic potentials in the evaluation of patients with air-bone gaps. Otol Neurotol 2012;33:1368-74

12 Sheykholeslami K, Murofushi T, Kaga K. The effect of sternocleidomastoid electrode location on vestibular evoked myogenic potential. Auris Nasus Larynx 2001;28:41-3

13 Valvassori GE, Clemis JD. The large vestibular aqueduct syndrome. Laryngoscope 1978;88:723-8

14 Van Wermeskerken GK, Dunnebier EA, Van Olphen AF, Van Zanten BA, Albers FW. Audiological performance after cochlear implantation: a 2-year follow-up in children with inner ear malformations. Acta Otolaryngol 2007;127:252-7

$15 \mathrm{Xu}$ XD, Zhang XT, Zhang Q, Hu J, Chen YF, Xu M. Ocular and cervical vestibular-evoked myogenic potentials in children with cochlear implant. Clin Neurophysiol 2015;126:1624-31

16 Sheykholeslami K, Schmerber S, Habiby Kermany M, Kaga K. Vestibular-evoked myogenic potentials in three patients with large vestibular aqueduct. Hear Res 2004;190:161-8

17 Merchant SN, Nakajima HH, Halpin C, Nadol JB Jr, Lee DJ, Innis WP et al. Clinical investigation and mechanism of airbone gaps in large vestibular aqueduct syndrome. Ann Otol Rhinol Laryngol 2007;116:532-41

18 Zhou G, Gopen Q, Poe DS. Clinical and diagnostic characterization of canal dehiscence syndrome: a great otologic mimicker. Otol Neurotol 2007;28:920-6

19 Minor LB. Clinical manifestations of superior semicircular canal dehiscence. Laryngoscope 2005;115:1717-27

Address for correspondence:

Dr Q Zhang,

157 Xiwu Road,

Xincheng District,

Xi'an 710004, China

Fax: +862987275892

E-mail: zhqent@163.com

Dr Q Zhang takes responsibility for the integrity of the content of the paper

Competing interests: None declared 